
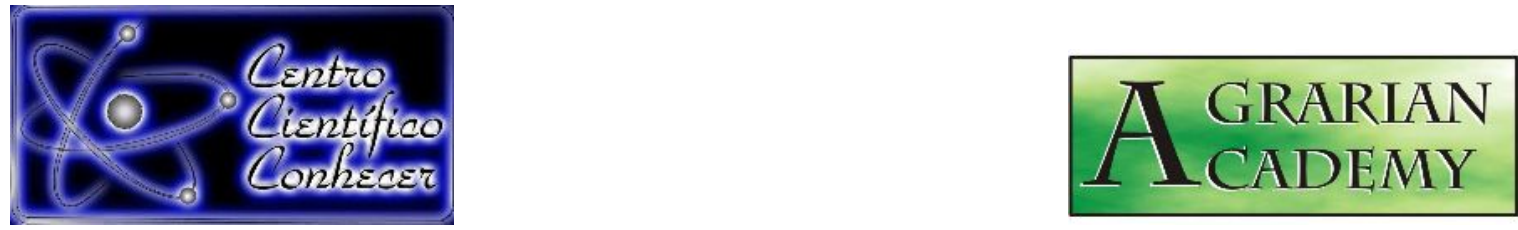

\title{
INFLUÊNCIA DA ADUBAÇÃO MINERAL NO DESENVOLVIMENTO INICIAL DE Schinus terebinthifolius Raddi
}

\author{
Oclizio Medeiros das Chagas Silva1, Lucas Santos Santana², Renato Luiz Grisi \\ Macedo ${ }^{3}$
}

1. Mestrando em Engenharia Florestal da Universidade Federal de Lavras, Departamento de Ciências Florestais, Lavras - Minas Gerais, Brasil. (omflorestal@hotmail.com)

2. Mestrando em Engenharia Agrícola na UFLA

3. Professor aposentado do Departamento de Ciências Florestais da UFLA

Recebido em: 19/11/2018 - Aprovado em: 14/12/2018 - Publicado em: 25/12/2018 DOI: 10.18677/Agrarian_Academy_2018B11

\begin{abstract}
RESUMO
O objetivo desse trabalho foi avaliar a influência da adubação mineral no desenvolvimento inicial da Schinus terebinthifolius Raddi. (aroeira pimenteira). $\mathrm{O}$ experimento foi conduzido no Centro de Desenvolvimento Científico e Tecnológico da UFLA - Fazenda Muquém. O delineamento experimental utilizado foi em blocos casualizados completos, com 12 repetições, em esquema fatorial 2x4 (uma espécie e uma duplicata e quatro doses de fósforo). Foram utilizadas quatro doses de fósforo (super fosfato simples) 0, 150, 300 e 450 gramas como adubação de base e como adubação de cobertura utilizou-se 70 gramas de NPK 20-00-20. Aos 60 e 300 dias pós-plantio, foram coletados dados de diâmetro a altura do solo, altura das plantas e área de copa para fins de análise do desenvolvimento inicial das plantas em campo. Os dados foram submetidos a análise de variância e as médias comparadas pelo teste de Scott e Knott (1974) a 5\% de significância. As dosagens de 300 e 450 gramas de fósforo contribuíram significativamente para o desenvolvimento da espécie Schinus terebinthifolius (aroeira pimenteira). As dosagens que mais favoreceram o crescimento dessa espécie foram de 300 a 450 gramas de $P$, sendo estas recomendadas para aplicação na fase inicial, considerando as condições desse estudo.
\end{abstract}

PALAVRAS-CHAVE: crescimento de plantas, parâmetros morfológicos, recomendação de fertilizantes.

\section{INFLUENCE OF MINERAL FERTILIZATION IN THE INITIAL DEVELOPMENT OF Schinus terebinthifolius Raddi}

\footnotetext{
ABSTRACT

The objective of this work was to evaluate the influence of mineral fertilization on the initial development of Schinus terebinthifolius Raddi. (aroeira pepper). The experiment was conducted at the Center for Scientific and Technological AGRARIAN ACADEMY, Centro Científico Conhecer - Goiânia, v.5, n.10; p. 1122018
} 
Development of UFLA - Fazenda Muquém. The experimental design was a complete randomized complete block, with 12 replications, in a 2x4 factorial scheme (one species and one duplicate and four doses of phosphorus). Four doses of phosphorus (single super phosphate) 0, 150, 300 and 450 grams were used as base fertilization and 70 grams of NPK 20-00-20 were used as cover fertilization. At 60 and 300 days after planting, data were collected on soil height, plant height and crown area for analysis of the initial development of the plants in the field. The data were submitted to analysis of variance and the means were compared by the Scott and Knott test (1974) at 5\% significance. The dosages of 300 e 450 grams of phosphorus contributed significantly to the development of the species Schinus terebinthifolius (aroeira pepper). The dosages that favored the growth of this species were 300 to 450 grams of $P$, and these were recommended for application in the initial phase, considering the conditions of this study.

KEYWORDS: fertilizer recommendation, morphological parameters, plant growth.

\section{INTRODUÇÃO}

A Schinus terebinthifolius Raddi. (aroeira pimenteira), é uma espécie originária da América do Sul, nativa do Brasil, Paraguai, Uruguai e leste da Argentina. É largamente distribuída pelo território brasileiro, estendendo-se desde Pernambuco até Rio Grande do Sul. É empregada na recuperação de áreas degradadas, utilizada como alimento, sendo também base para produtos medicinais, além de outros usos (SILVA-LUZ; PIRANI 2012).

Os solos brasileiros geralmente são pobres quimicamente e apresentam baixa fertilidade. Dentre os nutrientes o fósforo é um dos mais limitantes do crescimento vegetal na fase inicial (GRANT et al., 2001). Estudos referentes ao fornecimento de fósforo como adubação de base e aplicação de nitrogênio, fósforo e potássio (NPK) em cobertura são de grande importância para compreensão da função destes, no crescimento e desenvolvimento das espécies em campo (SCHUMACHER et al., 2004).

O fósforo e o nitrogênio são os nutrientes que geralmente limitam o crescimento de mudas na fase inicial de produção, uma vez que teores elevados desses elementos são altamente requeridos nos estádios iniciais para crescimento vegetativo da parte aérea e desenvolvimento do sistema radicular. O P é um dos nutrientes que merecem atenção para a produção florestal, pois a disponibilidade desse elemento, em condições naturais, é muito baixa. A adubação fosfatada, dessa forma, é prática imprescindível na condução de plantios florestais (VILAR; VILAR 2013).

Para a condução de plantios de espécies nativas, há uma carência de estudos sobre seus requerimentos nutricionais, podendo este comprometer o desenvolvimento das plantas em campo (SORREANO et al., 2012). O conhecimento acerca das exigências nutricionais deve ser considerado, buscando melhorar a concisão e quantificação de dosagens de adubação e também o custo financeiro do produtor (AQUINO et al., 2013; VIEIRA et al., 2017).

A adubação é parte essencial no crescimento de uma espécie. Vários estudos com essências florestais, principalmente para fins comerciais, têm demonstrado que a fertilização contribui para 0 aumento em produtividade, qualidade e o estabelecimento dos plantios florestais (CAMPOS et al., 2014). Porém, detalhamento sobre recomendações e exigências nutricionais principalmente para 
espécies nativas ainda são escassas e pouco encontradas na literatura. $O$ conhecimento do comportamento nutricional peculiar a cada espécie gera maior produtividade e menores impactos econômicos e ao meio ambiente (CARLOS et al., 2014; GONÇALVES et al., 2014).

A aplicação de fertilizantes pode favorecer o crescimento e melhorar a produção dessa espécie. Neste contexto, o presente trabalho teve como objetivo avaliar a influência da adubação mineral no crescimento inicial da aroeira pimenteira.

\section{MATERIAL E MÉTODOS}

O experimento foi implementado no ano de 2017, no Centro de Desenvolvimento Científico e Tecnológico da UFLA - Fazenda Muquém, situado no município de Lavras, Minas Gerais, sob as coordenadas Latitude $21^{\circ} 11^{\prime} 42.52$ 'S e Longitude 4459'21.50"O (Figura 1).
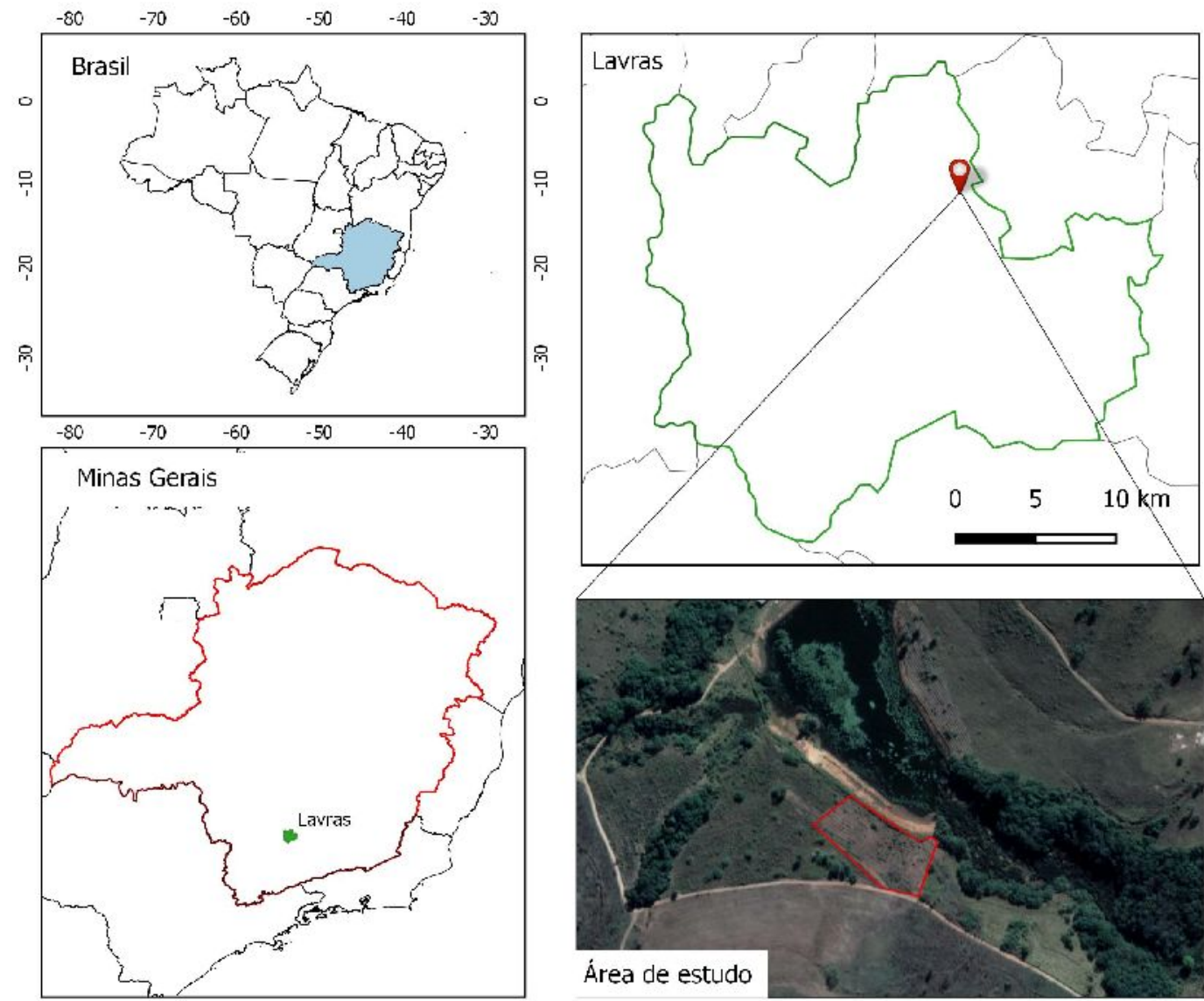

FIGURA 1. Mapa de localização da área onde o experimento onde foi conduzido.

De acordo com classificação climática de Köppen, o clima de Lavras é Cwa, temperado chuvoso (mesotérmico) com inverno seco e verão chuvoso, subtropical, sendo a temperatura do mês mais quente maior que $22^{\circ} \mathrm{C}$, com temperatura média de $19,9^{\circ} \mathrm{C}$, apresentando pluviosidade média anual de $1486 \mathrm{~mm}$ (ALVARES et al., 2013).

Para avaliar a fertilidade do solo da área, foram feitas amostragens de solo, nas profundidades de $0,0-0,2 \mathrm{~m}$ e $0,2-0,4 \mathrm{~m}$ e encaminhadas ao Laborátorio de 
Análises de Solo do Departamento de Agronomia da UFLA para posterior análises (Tabela 1).

TABELA 1. Características químicas do solo da área de instalação do experimento nas profundidades de 0,0-0,2m e 0,2-0,4m.

\begin{tabular}{|c|c|c|c|c|c|c|c|c|}
\hline Profundidade & pH & $\mathrm{Ca}$ & Mg & Al & $\mathrm{H}+\mathrm{Al}$ & SB & $\mathbf{t}$ & $\mathbf{T}$ \\
\hline $\mathbf{c m}$ & \multicolumn{8}{|c|}{ 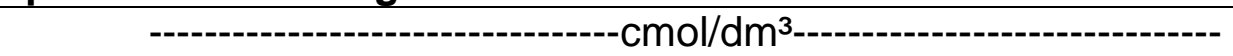 } \\
\hline $00-20$ & 6,3 & 3,22 & 0,75 & 0,06 & 1,82 & 4,2 & 4,26 & 6,02 \\
\hline $20-40$ & 5,8 & 2,79 & 0,67 & 0,05 & 3,17 & 3,61 & 3,66 & 6,78 \\
\hline \multirow[t]{2}{*}{$\mathbf{c m}$} & $\mathbf{P}$ & $\mathbf{K}$ & V & $\mathbf{m}$ & M.O & P-rem & $\mathrm{Zn}$ & B \\
\hline & \multicolumn{2}{|c|}{----mg/dm³---- } & \multicolumn{2}{|c|}{--------\%-------- } & $\mathrm{dag} / \mathrm{kg}$ & $\mathrm{mg} / \mathrm{L}$ & \multicolumn{2}{|c|}{$---m g / d^{3}$} \\
\hline $00-20$ & 0,68 & 90,87 & 69,82 & 1,41 & 1,92 & 32,13 & 1,99 & 0,18 \\
\hline $20-40$ & 0,62 & 59,03 & 53,26 & 1,37 & 1,68 & 30,54 & 1,09 & 0,14 \\
\hline
\end{tabular}

Para o plantio, as mudas foram fornecidas pelo Viveiro da Unidade da Usina Hidrelétrica Volta Grande, do município de Conceição das Alagoas, Minas Gerais. O delineamento experimental utilizado foi em blocos casualizados completos, com 12 repetições, em esquema fatorial 2x4 (uma espécie e uma duplicata e quatro doses de fósforo. A espécie utilizada foi Schinus terebinthifolius Raddi (aroeira pimenteira).

Foram utilizadas quatro doses de fósforo (super fosfato simples) 0, 150, 300 e 450 gramas como adubação de base. O superfosfato simples foi posto no fundo da cova e posteriormente foi feito o plantio das mudas em espaçamento $3 \times 1,5 \mathrm{~m}$. Aos sete meses foram aplicados como adubação de cobertura 70 gramas por cova de NPK 20-00-20, sendo esta adubação feita na aroeira (denominada de aroeira 1) e a duplicata (denominada de aroeira 2) foi utilizada como testemunha não sendo empregada adubação de cobertura.

Foram realizadas duas coletas de dados para fins de análises do desenvolvimento inicial das mudas em campo. Aos 60 dias após plantio, ocorreu a mensuração dos parâmetros biométricos diâmetro a altura do solo (DAS) com auxílio de um paquímetro digital $(\mathrm{mm})$ e altura das plantas $(\mathrm{H})$ utilizando-se vara graduada (cm), de acordo com Resende et al. (2015). Aos 300 dias, além da avaliação da altura e diâmetro, foram reunidos dados da área de copa (AC), com o auxílio de uma vara graduada $(\mathrm{cm})$, na qual foram coletadas duas medidas ortogonais, posteriormente calculada pela fórmula da elipse ( $\mathrm{AC}=\mathrm{a} \times \mathrm{b} \times \mathrm{m} / 4)$.

Para a compilação dos dados realizou-se a análise de variância e posteriormente as médias foram comparadas pelo teste de Scott e Knott (1974) a 5\% de significância. Para tal foi utilizado o programa Sisvar versão 5.6 (FERREIRA 2014).

\section{RESULTADOS E DISCUSSÃO}

Os valores correspondentes as avaliações dos parâmetros: diâmetro a altura do solo (DAS), altura das plantas $(\mathrm{H})$ e área de copa $(\mathrm{AC})$ referente as duas coletas estão demonstrados nas tabelas seguintes. 
TABELA 2. Efeito médio das espécies para o diâmetro à altura do solo $(\mathrm{mm})$ e altura das plantas $(\mathrm{cm})$ aos 60 dias após plantio.

\begin{tabular}{l|c|c|c|c}
\hline \multirow{2}{*}{ Espécies } & \multicolumn{5}{c}{ Doses de P em mg dm-3 } \\
\cline { 2 - 5 } & $\mathbf{0}$ & $\mathbf{1 5 0}$ & $\mathbf{3 0 0}$ & $\mathbf{4 5 0}$ \\
\hline \multicolumn{5}{c}{ DAS (mm) aos 60 dias } \\
\hline Aroeira 1 & $9,18 \mathrm{~b}$ & $12,70 \mathrm{a}$ & $14,35 \mathrm{a}$ & $14,46 \mathrm{a}$ \\
Aroeira 2 & $9,34 \mathrm{~b}$ & $12,42 \mathrm{a}$ & $12,56 \mathrm{a}$ & $12,87 \mathrm{a}$ \\
\hline \multicolumn{5}{c}{ Doses de P em mg dm-3 } \\
\hline \multirow{7}{*}{ Espécies } & $\mathbf{0}$ & $\mathbf{1 5 0}$ & $\mathbf{3 0 0}$ & $\mathbf{4 5 0}$ \\
\cline { 2 - 5 } & \multicolumn{5}{c}{ Altura (cm) aos 60 dias } \\
\hline Aroeira 1 & $53,08 \mathrm{c}$ & $67,70 \mathrm{~b}$ & $68,90 \mathrm{~b}$ & $80,08 \mathrm{a}$ \\
Aroeira 2 & $57,83 \mathrm{c}$ & $64,80 \mathrm{~b}$ & $67,08 \mathrm{~b}$ & $70,75 \mathrm{a}$ \\
\hline
\end{tabular}

Médias seguidas pela mesma letra não diferem significativamente entre si pelo teste de Skott-Knott ao nível de 5\% de probabilidade de erro.

Ao analisar o efeito das diferentes doses de fósforo, observou-se que a aplicação desse nutriente levou a ganhos significativos de crescimento em diâmetro das plantas quando comparado às plantas com dosagens zero de adubação (Tabela 2). Não houve diferença significativa entre as dosagens 150, 300 e $450 \mathrm{mg} \mathrm{dm}-3$ para esta variável tanto para a aroeira como para a duplicata.

Quando analisada a variável altura, notou-se que quanto maior a dosagem, maiores foram os ganhos. A dosagem de $450 \mathrm{mg} \mathrm{dm}-3$ de $\mathrm{P}$ foi a que mais contribui para o desenvolvimento em altura. Em contrapartida, a dosagem com zero de adubação diferiu estaticamente, sendo inferior as demais.

Avaliando o crescimento inicial de quatro espécies florestais, com diferentes níveis de adubação em área degradada, Sheer et al. (2017) constataram que a aroeira apresentou diâmetro estaticamente superior as demais, constatando assim que a espécie respondeu significativamente quando esta recebeu dosagens de adubação. O fósforo é um nutriente muito requerido pelas plantas na fase inicial e também este é aplicado devido aos baixos valores encontrados nos solos brasileiros.

Segundo Chiamolera et al. (2011) o maior crescimento em altura e diâmetro da aroeira também pode estar relacionado a adaptação, pois esta apresenta plasticidade fenotípica, podendo assim demonstrar maior tolerância ao ambiente onde foram inseridas.

TABELA 3. Efeito médio das espécies para o diâmetro à altura do solo $(\mathrm{mm})$, altura das plantas $(\mathrm{cm})$ e área de copa $\left(\mathrm{m}^{2}\right)$ aos 300 após plantio.

\begin{tabular}{c|c|c|c|c}
\hline \multirow{2}{*}{ Espécies } & $\mathbf{5}$ Doses de P em mg dm-3 \\
\cline { 2 - 5 } & $\mathbf{0}$ & $\mathbf{1 5 0}$ & $\mathbf{3 0 0}$ & $\mathbf{4 5 0}$ \\
\hline \multicolumn{5}{c}{ DAS (mm) aos $\mathbf{3 0 0}$ dias } \\
\hline Aroeira 1 & $30,24 \mathrm{Ab}$ & $38,44 \mathrm{Aa}$ & $43,31 \mathrm{Aa}$ & $41,43 \mathrm{Aa}$ \\
Aroeira 2 & $21,13 \mathrm{Bc}$ & $27,89 \mathrm{Bb}$ & $35,31 \mathrm{Bb}$ & $36,8 \mathrm{Bb}$ \\
\hline \multicolumn{5}{c}{ Doses de P em $\mathbf{~ m g ~ d m - 3}$} \\
\hline \multirow{2}{*}{ Espécies } & $\mathbf{0}$ & $\mathbf{1 5 0}$ & $\mathbf{3 0 0}$ & $\mathbf{4 5 0}$ \\
\cline { 2 - 5 } &
\end{tabular}

AGRARIAN ACADEMY, Centro Científico Conhecer - Goiânia, v.5, n.10; p. 1162018 


\begin{tabular}{|c|c|c|c|c|}
\hline \multicolumn{5}{|c|}{ Altura $(\mathrm{cm})$ aos 300 dias } \\
\hline Aroeira 1 & $169,33 \mathrm{Ab}$ & $196,33 \mathrm{Aa}$ & $201,36 \mathrm{Aa}$ & $221,45 \mathrm{Aa}$ \\
\hline Aroeira 2 & $136,86 \mathrm{Bc}$ & $150,33 \mathrm{Bb}$ & $171,09 \mathrm{Bb}$ & $194,58 \mathrm{Bb}$ \\
\hline \multirow{2}{*}{ Espécies } & \multicolumn{4}{|c|}{ Doses de P em mg dm-3 } \\
\hline & 0 & 150 & 300 & 450 \\
\hline \multicolumn{5}{|c|}{ Área de copa $\left(\mathrm{m}^{2}\right)$ aos 300 dias } \\
\hline Aroeira 1 & $0,42 \mathrm{Ac}$ & $0,88 \mathrm{Ab}$ & $1,49 \mathrm{Aa}$ & 1,54 Aa \\
\hline Aroeira 2 & $0,22 \mathrm{Bd}$ & $0,61 \mathrm{Bc}$ & $0,83 \mathrm{Bb}$ & $1,23 \mathrm{Bb}$ \\
\hline
\end{tabular}

Médias seguidas pela mesma letra não diferem significativamente entre si pelo teste de Skott-Knott ao nível de 5\% de probabilidade de erro.

Para a variável diâmetro na avaliação 300 dias, a aroeira 1 foi a espécie que mais expressou crescimento 169,33 196,33, 201,36 e 221,45 mm respectivamente. Entre as dosagens 150,300 e $450 \mathrm{mg} \mathrm{dm}-3$ não houve diferença significativa considerando o delineamento inicial. Quando se comparou a aroeira $1 \mathrm{com}$ a aroeira 2 , notou-se que houve diferença significativa, corroborando que a adubação de cobertura teve efeito direto no desenvolvimento dessa variável (DANTAS NETO et al., 2013). Dados semelhantes foram encontrados por Dias et al. (2016). Estes autores avaliaram diferentes dosagens de fósforo no desenvolvimento de espécies nativas, e embora com algumas variações, as maiores médias foram encontradas nas concentrações de adubo fosfatado entre 360 e $540 \mathrm{~g}$ de $\mathrm{P}$.

Quando analisada a altura das plantas a aroeira 1 foi superior a aroeira 2, apresentando maior crescimento. Considerando o delineamento inicial não houve diferença significativa entre as dosagens 150,300 e $450 \mathrm{mg} \mathrm{dm}-3$. Ao comparar entre espécie, observou-se que 0 crescimento em altura da aroeira 1 foi estaticamente superior ao da aroeira 2. A adubação de cobertura forneceu nitrogênio as plantas, sendo este elemento essencial para 0 crescimento vegetativo (SCHUMACHER et al., 2004).

Ao analisar a Tabela 3, notou-se que a espécie que mais desenvolveu área de copa foi a aroeira $1 \mathrm{com}$ média de 1,49 e 1,54 $\mathrm{m}^{2}$ para as dosagens de 300 e 450 mg dm-3 respectivamente. Para a dosagem com zero de adubação 0 desenvolvimento em copa foi estaticamente inferior aos tratamentos com adubação.

Em trabalho desenvolvido por Knapik et al. (2005) estes autores avaliaram o crescimento inicial de três espécies sob diferentes níveis de adubação, a Schinus terebinthifolius foi a que melhor respondeu a adubação, principalmente resposta ao tratamento que continha fósforo. Ao analisar a influência de diferentes doses de fósforo no crescimento do angico-vermelho (Parapiptadenia rígida), Schumacher et al. (2004) constataram que para a variável altura da parte aérea, a dose de $450 \mathrm{mg}$ $\mathrm{dm}-3$ de $\mathrm{P}$ foi a que apresentou os melhores resultados, ao passo que para as variáveis diâmetro e biomassa acima do solo, a dosagem recomendada foi de 360 mg dm-3 de P.

Souza et al. (2001), avaliando o estabelecimento de espécies na recuperação de uma área, constataram que a aroeira se apresentou como espécie promissora quanto ao crescimento médio em altura, em diâmetro do caule ao nível do solo e quanto à área de copa. Ao avaliarem o efeito de diferentes dosagens de fertilizante fosfatado, no crescimento de espécies florestais nativas, Dias et al. (2016) 
constataram que a dosagem de $360 \mathrm{~g}$ de $\mathrm{P}$ proporcionou a maior média, aos parâmetros avaliados para a aroeira.

Em estudo, analisando o efeito de frações fosfatadas em três espécies florestais, Valadares et al. (2015) constataram que o aumento da adubação fosfatada resultou em resposta quadrática positiva para altura e diâmetro do colo. Os máximos crescimentos em altura $(8,64 \mathrm{~cm})$ e em diâmetro do colo $(2,55 \mathrm{~mm})$ foram obtidos nas doses correspondentes a 278 e 328 mg dm-3 de $P$, respectivamente, semelhante as dosagens indicadas para a espécie Schinus terebinthifolius deste estudo.

De modo geral para as variáveis: diâmetro, altura e área de copa em média as aroeiras responderam de forma significativa as dosagens de fósforo aplicadas quando comparadas com tratamentos com zero de adubação, e quando realizou-se a aplicação de nutrientes em cobertura, a espécie do estudo teve comportamento superior quando comparada a testemunha.

\section{CONCLUSÕES}

A espécie Schinus terebinthifolius (aroeira pimenteira) expressou de forma significativa ao desenvolvimento dos parâmetros avaliados quando submetida as diferentes dosagens de adubação. Recomenda-se as dosagens de 300 a $450 \mathrm{mg}$ dm-3 por planta para favorecer o desenvolvimento dessa espécie em campo.

\section{REFERÊNCIAS}

ALVARES, C. A.; STAPE, J. L.; SENTELHAS, P. C.; DE MORAES, G. J. L.; SPAVOREK, G. Köppen's climate classification map for Brazil. Meteorologische Zeitschrift, v. 22, p. 711-728, 2013. Disponível em: <http://www.ingentaconnect.com/content/schweiz/mz/2013/00000022/00000006/art0 0008>. doi: https://doi.org/10.1127/0941-2948/2013/0507

AQUINO, L. A.; SILVA, F. D. B.; BERGER, P. G. Agronomic characteristics and nutritional status of irrigated sunflower cultivars. Revista Brasileira de Engenharia Agrícola e Ambiental, Campina Grande, v. 17, p. 551-557, 2013. Disponível em: $<$ http://www.scielo.br/scielo.php?script=sci_arttext\&pid=S1415-

43662013000500013>. doi: http://dx.doi.org/10.1590/S1415-43662013000500013

CAMPOS, C. N. S. Introdução à nutrição e adubação de plantas. In: PRADO, R. M.; WADT, P. G. S. (Ed.). Nutrição e adubação de espécies florestais e palmeiras. Jaboticabal: FCAV/CAPES, p. 9-26, 2014.

CARLOS, L.; VENTURIN, N.; MACEDO, R. L. G.; HIGASHIKAWA, E. M.; GARCIA, M. B. et al. Crescimento e nutrição mineral de mudas de pequi sob efeito da omissão de nutrientes. Ciência Florestal, Santa Maria, v. 24, n. 1, p. 13-21, 2014. Disponível em: $<$ http://www.scielo.br/scielo.php?pid=S198050982014000100013\&script=sci_abstract\&tlng=pt >.

CHIAMOLERA, L.; B.; ANGELO. A. C.; BOEGER, M. R. Crescimento e sobrevivência de quatro espécies florestais nativas plantadas em áreas com diferentes estágios de sucessão no reservatório Iraí-PR. Revista Floresta, Curitiba, v. 41, n 4, p. 765-778, 2011. Disponível em: < https://revistas.ufpr.br/floresta/article/view/25341/16979>. 
DANTAS NETO, J.; MACIEL, J. L.; ALVES, A. S.; AZEVEDO, C. A. V. DE; FERNANDES, P. D. et al. Teores de macronutrientes em folhas de goiabeira fertirrigadas com nitrogênio. Revista Brasileira de Engenharia Agrícola e Ambiental, v.17, p.962-968, 2013. Disponível em <http://dx.doi.org/10.1590/S141543662013000900008>

DIAS, I. M.; BARRETO, Í. D. de C.; FERREIRA, R. A. Efeito de dosagens de fertilizante fosfatado na determinação de volume ótimo de produção de mudas de espécies florestais. Scientia Agraria Paranaensis, Paraná, v. 15, n. 4, out./dez., p. 471-475, 2016. Disponível em: https://www.researchgate.net/profile/Roberio_Ferreira/publication/318115957. doi: http://dx.doi.org/10.18188/1983-1471/sap.v15n4p471-475

FERREIRA, D. F. Sisvar: a Guide for its Bootstrap procedures in multiple comparisons. Ciência agrotécnica. [online]. vol. 38, n. 2, 2014 [citado 2015-10-17], pp. 109-112. Disponível em <http://dx.doi.org/10.1590/S1413-70542014000200001>.

GRANT, C. A.; FLATEN, D.N.; TOMASIEWICZ, D.J.; SHEPPARD, S. C. A importância do fósforo no desenvolvimento inicial da planta. Potafos - Associação Brasileira para Pesquisa da Potassa e do Fosfato. Informações Agronômicas, 95: 1-5. 2001. Disponível em: <http://www.ipni.net/publication/iabrasil.nsf/0/43C5E32F5587415C83257AA30063E620/\$FILE/Page1-5-95.pdf>

KNAPIK. J. G.; ALMEIDA, L. S.; FERRARI, M. P.; OLIVEIRA, E. D.; NOGUEIRA, A. C. Crescimento inicial de Mimosa scabella Benth., Schinus terebinthifolius Raddi e Allophylus edulis (St. Hil) Radl. sob diferentes regimes de adubação. Boletim. Pesquisa Florestal, Colombo, v. 3, n. 51, p. 33-44, 2005. Disponível em $<$ https://www.google.com.br/search?q=KNAPIK>.

RESENDE, L. A.; PINTO, L. V. A.; SANTOS, E. C.; SILVA S. Crescimento e sobrevivência de espécies arbóreas em diferentes modelos de plantio na recuperação de área degradada por disposição de resíduos sólidos urbanos. Revista Árvore, v. 39, p. 147-157, 2015. Disponível em: <http://www.scielo.br/scielo.php?pid=S010067622015000100014\&script=sci_abstrac t\&tlng=pt>. Doi: http://dx.doi.org/10.1590/0100-67622015000100014.

SCHUMACHER, M. V.; CECONI, D. E.; SANTANA ARRUDA, CEDINARA. Influência de diferentes doses de fósforo no crescimento de mudas de angico-vermelho (Parapiptadenia rigida (Bentham) Brenan). Revista Árvore, Viçosa, v. 28, n. 1, p. 149-155, 2004. Disponível em: http://www.redalyc.org/articulo.oa?id=48828119.

SCOTT, A. J.; KNOTT, M. A cluster analysis method for grouping means in the analysis of variance. Biometrics, Washington, v. 30, n. 3, p. 507-512, 1974. Disponível em: <https://www.ime.usp.br/ abe/lista/pdfXz71qDkDx1.pdf>.

SHEER, M. B.; CARNEIRO, C.; BRESSAN, O. A.; Dos SANTOS, K, G. Crescimento inicial de quatro espécies florestais nativas em área degradada com diferentes níveis de calagem e adubação. Floresta, Curitiba, PR, v. 47, n. 3, p. 279-287, 2017. 
Disponível em: https://revistas.ufpr.br/floresta/article/view/41973. doi: http://dx.doi.org/10.5380/rf.v47i3.41973.

SILVA-LUZ CL, PIRANI JR. Anacardiaceae in Lista de Espécies da Flora do Brasil Jardim Botânico, Rio de Janeiro, 2012. Disponível em: <http://floradobrasil.jbrj.gov.br/2012/index>.

SORREANO, M. C. M.; RODRIGUES, R. R.; BOARETTO, A. E. Guia de nutrição para espécies florestais nativas. São Paulo: Oficina de Textos, 2012, 254 p.. Disponível em: <https://www.ofitexto.com.br/livro/guia-de-nutricao-para-especiesflorestais-nativas/>.

SOUZA A.DE, P.; VENTURIN, N.; MACEDO G. R. L.; ALVARENGA N. M. I.; SILVA F.DA, V. Estabelecimento de espécies arbóreas em recuperação de área degradada pela extração de areia Cerne, v. 7, n. 2, p. 43-52, 2001. Disponível em: <http://www.redalyc.org/articulo.oa?id=74470205>.

VALADARES, S. V.; DA SILVA, L. F.; VALADARES, R. V.; FERNANDES, L. A.; NEVES, J. C. L et al. Plasticidade fenotípica e frações fosfatadas em espécies florestais como resposta à aplicação de fósforo. Revista Árvore, v. 39, n.. 2, p. 225232. 2015. Disponível em: <http://www.redalyc.org/articulo.oa?id=48839479002>. doi: http://dx.doi.org/10.1590/0100-67622015000200002

VIEIRA, C. R.; WEBER, O. L dos SANTOS.; SCARAMUZZA, J. F. Influência da adubação NPK no crescimento inicial de Tectona grandis L. f. em campo. Revista. Instituto Florestal. v. 29 n. 1 p. $57-69$ jun. 2017. Disponível em: http://dx.doi.org/10.24278/2178-5031.201729104.

VILAR, C. C. \& VILAR, F. C. M. Comportamento do fósforo em solo e planta. Campo Digital (Faculdade Integrado de Campo Mourão), v.8, p. 37-44, 2013. Disponível em: $\quad<$ https://pt.scribd.com/document/249732944/COMPORTAMENTO-DO FOSFORO-EM-SOLO-E-PLANTA>. 